\title{
Rapidly Interpreting UV-optical Light Curve Properties Using a "Simple" Modeling Approach
}

\author{
Janie De La Rosa ${ }^{1}$, Pete Roming ${ }^{1,2}$ (1), and Chris Fryer ${ }^{3,4,5}$ (1) \\ ${ }^{1}$ Department of Physics and Astronomy, University of Texas at San Antonio, San Antonio, Texas, 78249, USA \\ ${ }_{3}^{2}$ Southwest Research Institute, San Antonio, Texas, 78228, USA \\ ${ }^{3}$ Los Alamos National Laboratory, Los Alamos, NM 87545, USA \\ ${ }^{4}$ Department of Physics and Astronomy, University of New Mexico, Albuquerque, NM 87131, USA \\ ${ }^{5}$ Steward Observatory, The University of Arizona, Tucson, AZ 85721, USA \\ Received 2017 August 3; revised 2017 October 9; accepted 2017 October 10; published 2017 November 27
}

\begin{abstract}
Core-collapse supernovae (CCSNe) have very distinct observational properties that depend on the composition of the progenitor star, the dynamics of the explosion mechanism, and the surrounding stellar wind environment. In recent years, due to the uncertainty behind the type of massive star that evolves into different types of core-collapse events, there has been an increase in core-collapse supernova surveys aiding the advancement of numerical supernova simulations that explore the properties of the star before the explosion. Observationally, the unpredictable nature of these events makes it difficult to identify the type of star from which the CCSNe subtype evolves, but the issue from a theoretical standpoint relies on a gap in our current understanding of the explosion mechanism. The general light curve properties of CCSNe (rise, peak, and decay) by subtype are diverse, but appear to be homogeneous within each subtype, with the exception of Type IIn. Simplified SN models can be processed quickly in order to explore the properties of the progenitor star along with the explosion mechanism and circumstellar medium. Here, we present a suite of SN light curve models presented using a 1-temperature, homologous outflow light curve code. The SN explosion is modeled from shock breakout through the ultimate uncovering of the nickel core. We are able to rapidly explore the diversity of the SN light curves by studying the effects of various explosion and progenitor star parameters, including ejecta mass, explosion energy, shock temperature, and stellar radii using this "simple" calculation technique. Furthermore, we compare UV and optical modeled light curves to Swift UVOT IIn observations to identify the general initial conditions that enable the difference between SN 2009ip and SN 2011ht light curve properties. Our results indicate that the peak light curve is dominated by the shock temperature and explosion energy, whereas the shape depends on the mass of the ejecta and the explosion energy. Based on this modeling approach, the comparison SN light curves are a product of processes occurring after shock breakout, but before ${ }^{56} \mathrm{Ni}$ decay. Therefore, the energy from nickel decay does not play a major role in the light curves of these explosions. In general, the diversity between SN 2009ip and SN $2011 \mathrm{ht}$ can be explained by the differences in the outer ejecta mass and the explosion energy.
\end{abstract}

Key words: stars: massive - supernovae: general

\section{Introduction}

Observations of core-collapse supernovae (CCSNe) have provided a general classification system that is based primarily on the presence of hydrogen in their spectra. A Type $\mathrm{Ib} / \mathrm{Ic}$ spectrum reveals no hydrogen, while a Type II SNe spectrum displays prominent hydrogen lines. Type II SNe are further classified based on their light curve properties, and the hydrogen profile in their spectra. Type IIn SNe reveal a narrow hydrogen component, while Type IIP and Type IIL SNe lack this feature (Schlegel 1990; Filippenko 1997). For Type II SNe, the light curve difference relies on the plateau region observed in Type IIP light curves, which could be an indication of the variation in the amount of hydrogen remaining in the stellar envelopes of Type IIP and Type IIL events.

Information about the progenitor star is primarily inferred from their spectral profiles. For stars that have lost their hydrogen/helium envelope prior to explosion, it is assumed that the star experienced mass-loss through a binary interaction or through a strong stellar wind. Hydrogen-free (type $\mathrm{Ib} / \mathrm{Ic}$ ) supernovae $(\mathrm{SNe})$ are the result of a massive star that has lost a tremendous amount of mass long before the explosion, leaving the progenitor star stripped from its hydrogen envelope, and in the case of Type Ic the helium envelope has been stripped as well. Recently, there has been an ongoing debate on whether or not Type Ic progenitors are truly helium-free. Modeling results from Dessart et al. (2012) indicate Type Ib and Ic could have the same progenitor star and the traces of helium could be hidden in Type Ic spectra due to low levels of Ni-56 mixing. Based on these results, Liu et al. (2016) readdressed the hidden helium issue, demonstrating a helium-free Type Ic progenitor due to helium burning into oxygen, as indicated by Frey et al. (2013) for stars greater than $20 M_{\odot}$. On the other hand, hydrogen-rich (type II) $\mathrm{SNe}$ progenitors maintain their hydrogen envelopes, and the distribution and amount of hydrogen creates the diversity among Type II SNe. Type II $\mathrm{SNe}$ with low hydrogen ejecta densities decline more rapidly, while the brighter maximum luminosity achieved by Type IIL may be the result of a larger radius or higher explosion energy (Blinnikov \& Bartunov 1993; Moriya et al. 2016; Valenti et al. 2016).

Before the characterization of Type IIn, Arnett (1980) pioneered an analytic solution for Type II SNe based on the explosion and shock emergence theories used in previous numerical models. These analytic models provided the framework needed to test several explosion theories based on observational features, and the ability to explore the physical 
parameter space of the $\mathrm{SN}$ environment without being computationally expensive. In order to understand the correlation between Type II light curve behavior and expanding SN material, Chevalier (1992) used an alternative analytic modeling approach that explored the effects associated with the interaction between the expanding stellar material and the surrounding stellar wind medium. Therefore, many recent analytic studies that explore the effects of additional physical processes, i.e., shocks, radioactive decay, and recombination, are derivations of these pioneering works. Chatzopoulos et al. (2012) presents SN ejecta-CSM interaction light curve models powered by forward and reverse shocks to produce characteristic IIn light curves. Depending on CSM density and progenitor star parameters, these SN ejecta-CSM interaction models can also reproduce the rapid linear decay observed in IIL. As a result, the difference in these subtypes could rely on the optical depth of the CSM, as the narrow component in IIn spectra would be the result of SN ejecta-CSM interactions with a higher optical depth than that of Type IIL (Chatzopoulos et al. 2012). SN spectral observations indicate that the type of progenitor star that evolves into a IIn has undergone serious mass-loss prior to core-collapse, while maintaining its hydrogen envelope (e.g., Gal-Yam et al. 2007; Smith et al. 2011; Margutti et al. 2013). Focusing on postmaximum light curve decline to constrain SN ejecta and CSM properties, Moriya et al. (2013) produced CSM-interactionpowered light curve models aimed at IIn SN by assuming the SN ejecta interacts with a dense circumstellar medium revealing LBV mass-loss rates from a $10 M_{\odot}$ ejecta with explosion energies greater than $10^{52} \mathrm{erg}$.

For IIn SNe, using light curve characteristics to arrive at a unified IIn model creates a problem due to inconsistencies in IIn light curve behavior, making it difficult to pinpoint the explosion process responsible for IIn light curve features without early shock breakout observations. In order to identify which part of the explosion process plays a role in producing typically observed IIn light curve trends, we demonstrate how the different phases of the explosion affect light curve properties. We are searching for which initial conditions influence the diversity within the IIn subclass, along with a general understanding of possible IIn progenitor star properties and explosion dynamics. We begin by simulating the physical properties of the progenitor star and the explosion dynamics based on first principles. The fast-running capabilities of the simple models presented provides the ability to explore the stellar and explosion parameter space. By varying the input stellar and explosion parameters, we explore the possibility of degenerate cases, and analyze which parameters affect which light curve characteristics: light curve shape, rise and decay, and peak luminosity. In order to determine the general properties of the progenitor star and explosion, the grid of models is compared to Swift's (Gehrels et al. 2004; UVOT Roming et al. 2005) observed IIn SNe in search of a range of possible pre-explosion parameters. Identifying these general properties will provide insight into which explosion processes are responsible for the main light curve and what influences the observational diversity within the IIn subclass.

In Section 2, we describe our model and compare our technique to previous modeling techniques. In Section 3, we present the tested parameters, break down the anatomy of the modeled light curves, and describe the different modeled SN explosion phases. In Section 4, we present the modeled blackbody luminosities of the simulated SN explosions with the initial parameter described in the previous section. In Section 5, we present the two IIn SNe observed by UVOT and compare the peak luminosities and light curve shapes to our suite of models. In Section 6, we analyze UV and optical light curves of a subset of models in comparison to SN 2009ip and SN 2011ht. In the Sections 7 and 8, we discuss the results from this simple semi-analytical modeling technique and summarize this work and future work.

\section{Computational Methods}

A broad suite of methods has been used to distinguish the characteristics of core-collapse supernovae from supernova light curves. These methods range from analytic approaches that focus on specific aspects of the supernova emission (Arnett 1980, 1982; Chevalier 1992; Arnett et al. 2016), to fullscale radiation hydrodynamics calculations (Fryer 2009; Fryer et al. 2010; Bersten et al. 2011; Frey et al. 2013; Morozova et al. 2015; Kozyreva et al. 2017). Most of the analytic methods make simplifying assumptions. For example,Arnett et al. (2016), building on the earlier works Arnett (1980, 1982), assumes that ${ }^{56} \mathrm{Ni}$ decay powers the light curve at its peak (a valid assumption for type Ia supernovae) and that the opacity is constant. Similarly, the Chevalier (1992) approach assumed a pressure profile set by the shock, defining the luminosity as the diffusion of this energy moving outward, again assuming a constant opacity. The full-scale radiation hydrodynamics calculations do not have to make these simplifying assumptions, but suffer from resolution issues, particularly at the photosphere (Frey et al. 2013).

Here, we use a simple, explicit 1-temperature diffusion code on top of a homologous outflow, where the velocity of the ejecta is proportional to its initial radius. The 1-temperature model assumes that matter and radiation can be described by the same temperature. In these assumptions, the opacity can be described by a single value. We typically use the electron scattering value that, for core-collapse supernovae, is reasonably accurate for radiation flow. This 1-temperature assumption does have limitations, especially for the higher energies at the photosphere. Beyond the photosphere, we assume that photon losses quickly radiate away the energy in the zone using an exponential decay time, until the temperature drops below a critical value beyond which we assume no further radiation is emitted from this region. The energy deposition from ${ }^{56} \mathrm{Ni}$ is assumed to be in situ, and in our default case, the ${ }^{56} \mathrm{Ni}$ is assumed to be located in the innermost ejecta.

Our approach lies somewhere in between analytic and detailed calculations. We implement shock heating through an analytic derivation similar to that of Chevalier (1992), where our temperature profile can be set to the pressure profile used in his calculations. But we also include ${ }^{56} \mathrm{Ni}$ decay. We explicitly model the radiation diffusion, modeling the light curves. The strength of this method is that it is faster than more sophisticated implementations, which allows us to run large suites of models. At the same time, it is easier to include additional physics in a parametric way, studying the effects of opacity variations, shock heating, etc., as well as parameterizing the initial conditions for full suites. By assuming a homologous expansion and a 1-temperature diffusion algorithm, our simulations can serve as a bridge between the full-scale radiation hydrodynamic calculations and the analytic light curve results. 


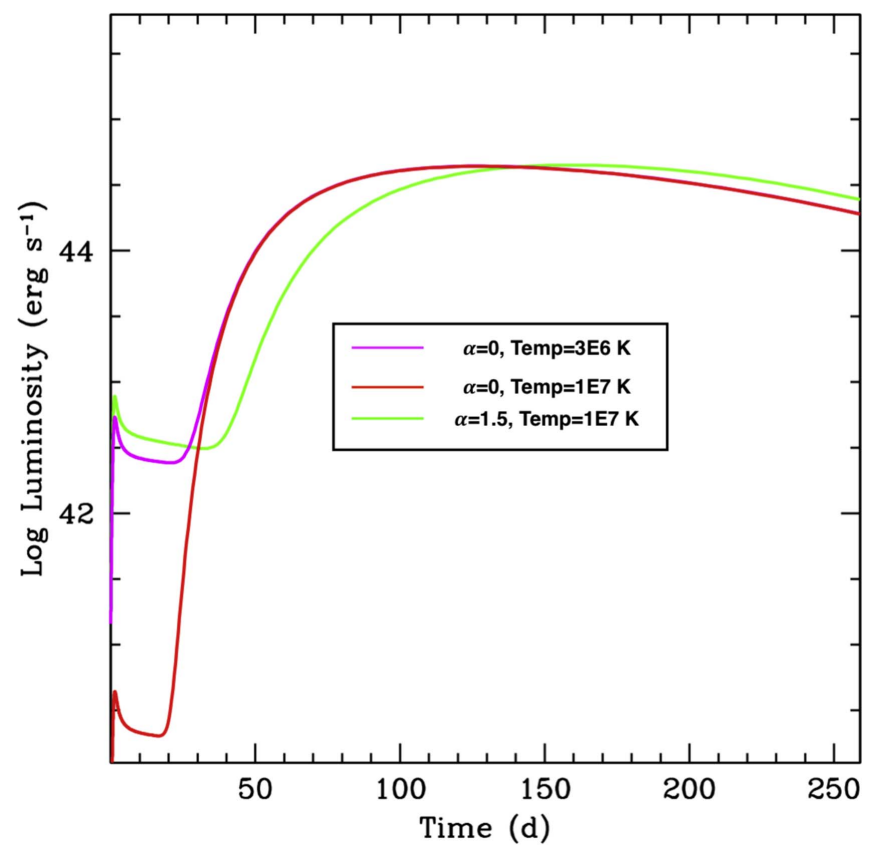

Figure 1. Luminosity vs. time for three pair-instability models based on the He130 model from Kozyreva et al. (2017). We have altered the power in the density profile from 0 (magenta, red) to 1.5 (green). We have also varied the initial temperature from $3 \times 10^{6} \mathrm{~K}$ to $10^{7} \mathrm{~K}$ (red, green). While the temperature determines the shock breakout, the ${ }^{56} \mathrm{Ni}$ and density distribution determine the rest of the light curve. Although the different codes in Figure 9 from Kozyreva et al. (2017) produce different results, the range of results agrees well with the simulations presented here.

\subsection{Code Validation}

For those models with detailed descriptions of the initial conditions, we can compare our method to past work. In Arnett et al. (2016), we found good agreement between analytic type Ia models and our simulations. We have also compared to the pair-instability models of Kozyreva et al. (2017). Figure 1 shows luminosity versus time for three different models, varying the density profile and temperature for the He130 model in Kozyreva et al. (2017). Our simulation better captures the shock breakout than some of the detailed transport calculations, and the breadth of light curve results compares well with the range of results from the different codes in the Kozyreva et al. (2017) study.

One of the problems with most simulations of supernova light curves is resolving the photosphere, and our models are no different. Most of our models use 2000 zones. Although this resolution is more resolved than many of the simulations published in the literature, this is well under the resolutions that Frey et al. (2013) argued were needed to truly resolve the photosphere. To determine the errors caused by our low resolution, we conducted a resolution study, studying the results for the four resolutions shown in Figure 2. As we increase the resolution, the primary light curve peak occurs earlier. As we shall see, the time of the peak depends on both the ejecta mass and velocity. As such, quantitative interpretations of the results depend on the resolution.

\section{Parameter Space and the Anatomy of a Light Curve}

In this paper, four properties of the progenitor star and explosion characteristics are varied: ejecta mass, stellar radius, explosion energy, and initial shock temperature. The input

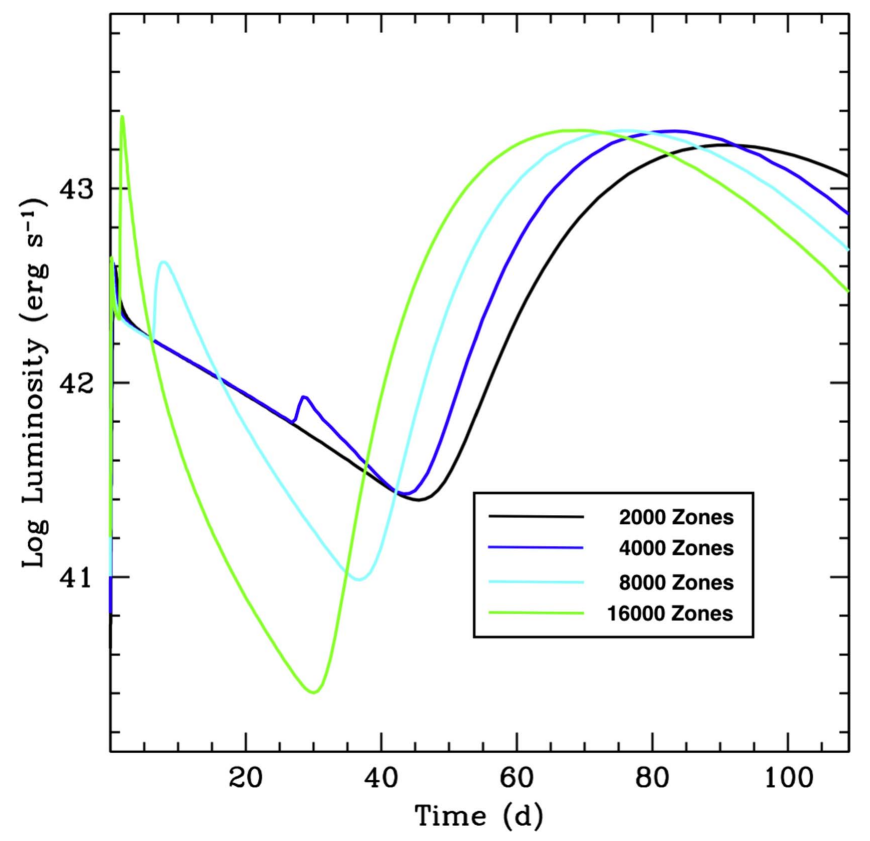

Figure 2. Luminosity vs. time for an $8 M_{\odot}$ ejecta mass, $5 \times 10^{51}$ erg explosion with $0.1 M_{\odot}$ for ${ }^{56} \mathrm{Ni}$ and a stellar radius of $5 \times 10^{12} \mathrm{~cm}$. The simulation shows four different spatial resolutions (three doublings from the original spacing) ranging from 2000 equally spaced zones (black) to 16,000 equally spaced zones (green). The higher the resolution, the sharper the breakout peak and the earlier the light curve.

values for each varied parameter tested are shown in Table 1. By sweeping the parameter space, we can analyze how each parameter affects the output luminosities, temperature, and expanding radius. Based on the appearance of a third peak in our preliminary results, we extended our simulation time frame from 150 days after explosion to 300 days. The extended time resolved the second light curve peak for many of the modeled dense stars. All of the simulations reveal a prominent first peak during the first 150 days of explosion. During the first 300 days, the less energetic models, combined with denser ejecta, fail to reveal the second light curve peak. The production of the third curve within 300 days depends on the ejecta mass and explosion energy.

Figure 3 shows how the physical properties of the explosion evolve based on the explosion energy for $15 M_{\odot}$ ejecta, with a $1 \times 10^{13} \mathrm{~cm}$ stellar radius, and an initial shock temperature of $1 \times 10^{6} \mathrm{~K}$. The expansion of the outermost layer produces the first light curve. The second re-brightening occurs as the optical depth $\tau$ of this outermost layer decreases, and the inner ejecta layers are responsible for shaping the second light curve peak. The third peak and smallest rise in the light curve occurs as the ${ }^{56} \mathrm{Ni}$ core is exposed. From our set of models, the transition from the first light curve to the second depends on the density of the outer layer, and the energy driving the explosion. Figure 3 shows how the energy of the explosion affects the structure of the first and second light curves. A higher explosion energy results in a shorter and brighter first peakduration, and a rapid transition from first to second peak, relative to the less energetic explosions. The radius of the highest explosion energy expands quickly, causing the ejecta of the outer mass zone to become optically thin earlier in the explosion process than it would for explosions with a lower expansion velocity. The higher explosion energy results in a higher blackbody temperature, and thus a brighter SN light 
Table 1

Model Parameters

\begin{tabular}{|c|c|c|c|c|c|c|c|}
\hline \multirow{2}{*}{$\begin{array}{l}\text { Variables } \\
\text { Radius }(\mathrm{cm})\end{array}$} & \multicolumn{7}{|c|}{ Parameters } \\
\hline & $8 \times 10^{12}$ & & $1 \times 10^{13}$ & & $3 \times 10^{13}$ & & $5 \times 10^{13}$ \\
\hline Energy (erg) & $1 \times 10^{50}$ & & $1 \times 10^{51}$ & & $5 \times 10^{51}$ & $\ldots$ & \\
\hline Temperature $(\mathrm{K})$ & $8 \times 10^{5}$ & & $1 \times 10^{6}$ & & $2 \times 10^{6}$ & & $3 \times 10^{6}$ \\
\hline Ejecta Mass $\left(M_{\odot}\right)$ & 10 & 15 & 20 & 25 & 35 & 45 & 60 \\
\hline
\end{tabular}

curve, but also displays a quick drop in temperature during the first peak as opposed to the second peak. As the outermost ejecta rapidly expand and cools, they reach a constant optical depth, initiating the homologous expansion of the inner ejecta layers that shape the second light curve peak. As we assume, photon losses quickly radiate away energy and temperatures drop rapidly beyond the outer photospheric layer, accounting for the high optical depth noise level for emission occurring beyond the photosphere. We define and analyze these distinct light curve peaks in the following sections for various progenitor star and explosion conditions in order to understand the role these properties play in shaping IIn light curve properties.

\subsection{Description of SN Phases}

In Figure 4, we introduce four SN explosion phases as the ejecta expand over time. The modeled mass density is used to demonstrate how the mass ejecta evolve over time. The physical properties of the $\mathrm{SN}$ model in Figure 4 represent $15 M_{\odot}$ ejecta, with a $1 \times 10^{13} \mathrm{~cm}$ stellar radius, and an initial shock temperature of $1 \times 10^{6} \mathrm{~K}$, for an explosion energy of $1 \times 10^{51} \mathrm{erg}$. The density profile before explosion at $t=0$ is shown in Phase 0. The four explosion phases shown are the optically thick expansion phase, shock breakout, the photospheric phase, and the receding photosphere to describe the evolution of the mass layer producing emission over time.

\subsubsection{Initial Conditions}

The first phase represents the conditions of the star before explosion, which demonstrates the initial density profile of the stellar envelope. Inside of the star, the pre-explosion density profile of the star is $\rho \propto\left(R_{\text {star }}^{3}-r_{i}^{3}\right)^{\alpha}$, where $R_{\text {star }}$ is the radius of the star, $r_{i}$ is the distance from the center of the star, and $\alpha=2$. We assume flat post-shock heating temperatures, which then define the internal energy of the shock $E_{\text {int }} \propto a T^{4}$. The velocity of the ejected stellar envelope is $v \propto \sqrt{E_{\exp } / M_{\text {ejecta }}}$, where $E_{\text {exp }}$ is energy of the explosion, and $M_{\text {ejecta }}$ is the mass of the ejecta at $r_{i}$. Therefore, the outermost mass layer expands at an initial velocity $v_{0}$ determined by the $M_{\text {ejecta }}$ enclosed in the outermost radial zone of the star. The initial mass density of $15 M_{\odot}, 1 \times 10^{13} \mathrm{~cm}$, the shock heating the ejecta to $1,000,000 \mathrm{~K}$, and the explosion energy of $10^{51} \mathrm{erg}$ are shown in Phase 0 of Figure 4. The core of the star makes up 10\% of the mass of the ejecta. The density profile of each layer is used to trace the density evolution over radius and time after the explosion for the following phases.

\subsubsection{Pre-shock Breakout}

This phase begins as the outer mass layer begins to travel away from the initial radius of the star, and is depicted as Phase 1 in Figure 4. As the outer ejecta layer expands and diffuses, the mass density and optical depth decrease. For this particular set of initial conditions, the pre-shock breakout phase lasts 5 days and the density of the outermost layer has dropped 4 mag by the time of the shock breakout. During this optically thick expansion phase, the ejecta travel from the initial radius of the star at $v_{0}$, photons are trapped, and there is no radiation being radiated away, $L_{\mathrm{rad}}=0$. The temperature of the ejecta decreases as a function of the expanding radius, while the internal energy of the explosion remains constant due to the lack of radiation leaving the system during this time. As the outer layer continues to expand, the density of the ejecta continues to decrease until the material is optically thin enough for the photons to escape. This relationship between the density of the ejecta and the optical depth determines the ability to release photons. The moment photon release occurs signifies the end of the optically thick expansion phase and the beginning of the shock breakout phase. The outer boundary of this phase resembles photospheric conditions as the outer ejecta density transitions into a less opaque region.

\subsubsection{Shock Breakout}

During shock breakout, the diffusion of photons is driven by the outermost ejecta shell as it continues to drop in opacity as the material is rushed away at $v_{0}$. The outer ejecta layer now has now traveled through the first photospheric region, as the optical depth is low enough to allow photons to pass through, producing the first peak in our modeled light curves. During this phase, the luminosity of the ejecta is dependent on the optical depth of the ejecta and is defined by $L_{\mathrm{rad}}=$ $4 \pi \sigma r_{i}^{2} T_{i}^{4} e^{-\tau_{i}}$, where $L_{\mathrm{rad}}$ is the luminosity radiated away, $\sigma$ is the Stefan-Boltzmann constant, $T_{i}$ is the temperature at radius $r_{i}$, and $\tau_{i}$ is the optical depth at radius $r_{i}$. The optical depth of the outermost layer continues to drop substantially, until it can no longer be the source of photon production. The following inner layer becomes responsible for photon emission. In this model, only the properties and structure of the outermost layer influence the characteristics of the shock breakout. The outer radius of Phase 2 in Figure 4 demonstrates the end of the shock breakout phase where the outer layer has expanded to 100 times the size of the initial radius and its density has dropped by 6 mag.

\subsubsection{Receding Photosphere and ${ }^{56} \mathrm{Ni}$ decay}

The second light curve emission is produced from the expansion of the inner stellar material. The outer photospheric boundary is interpreted as being located at the point where the outer layer becomes too optically thin to produce photon emission. This transition causes a re-brightening demonstrated in Figure 3 as the second peak in the light curve. The following phases describe the evolution of the second peak based on our modeling approach. The luminosity during this phase is defined as $L_{\mathrm{rad}}=4 \pi \sigma r_{i}^{2} T_{i}^{4}$. A second light curve peak occurs during this phase and the peak of this light curve occurs right before the explosion pushes the inner stellar material to its furthest 

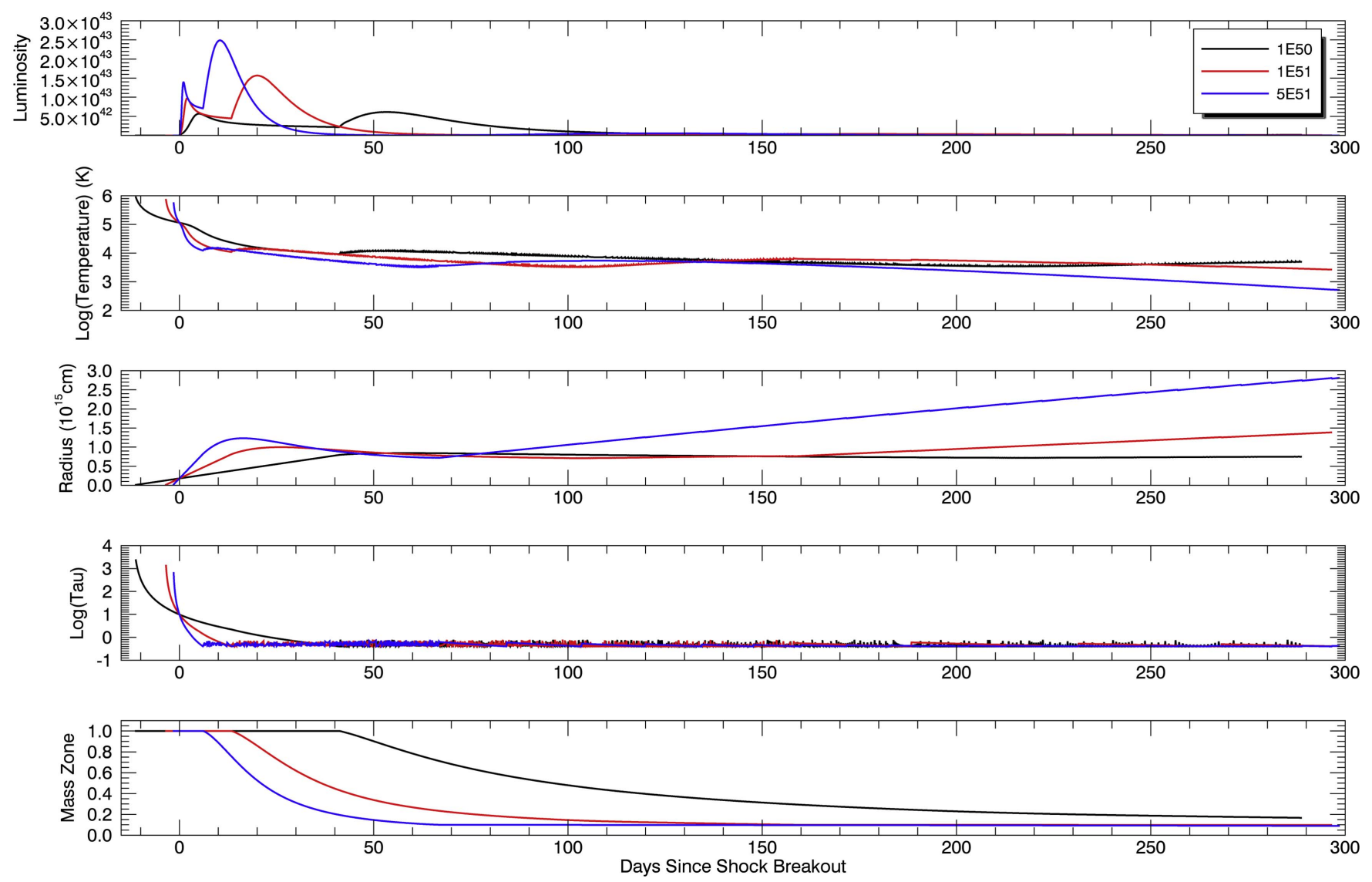

Figure 3. Luminosity, temperature, radius, optical depth, and mass zone vs. time for a $10 M_{\odot}$, initial radius of $1 \times 10^{13} \mathrm{~cm}$, and shock temperature of $1,000,000 \mathrm{~K}$, for three different explosion energies, $1 \times 10^{50} \mathrm{erg}, 1 \times 10^{51} \mathrm{erg}$, and $5 \times 10^{51} \mathrm{erg}$. The higher the explosion energy, the brighter the SN event. A mass zone of 1.0 corresponds to the outer mass layer. The higher the explosion energy, the faster the inner ejecta are exposed.

interaction point. During the peak luminosity, the expansion velocity slows down, and the photospheric radius remains momentarily constant, until it begins to recede inward toward the core. The transition to a receding photosphere occurs several days after the peak luminosity, during the decay portion of the second peak, and before the third peak is revealed. The decay of the second light curve corresponds to the interaction region moving inward toward the material originating from the stellar core. This process continues until each stellar layer has been exposed, including the region of ${ }^{56} \mathrm{Ni}$ located at the center of the star. The ${ }^{56} \mathrm{Ni}$-induced peak is revealed only in highenergy and low-mass models, as the third peak for denser and low-energy simulations is revealed to be beyond our 300-day time frame.

\section{Effects of Varied Parameters on Blackbody Luminosities}

In the previous sections we demonstrated the effects of varying the explosion energy. Therefore, in Figure 5, we explore how varying ejecta mass, stellar radius, and shock temperature alter blackbody luminosities for an explosion energy of $1 \times 10^{51} \mathrm{erg}$. We swept the parameter space discussed in Section 3 in order to identify a general relationship between the initial conditions of our simulations and the general properties of these simple blackbody luminosity models. We have associated the initial peak with the shock breakout phase, the second re-brightening corresponds to the photospheric material expanding and ultimately receding, and the third fairly resolved peak is the result of the nickel decay.
The first two curves are fully resolved within the first 300 days of the explosion, while the third peak makes an appearance for the explosions with a lower ejecta mass and a higher explosion energy. Based on a mass-radius relationship, our results indicate that stars with lower density profiles (low mass, large radius) produce brighter peak luminosity. The effect of the initial radius of the star causes minor variations in the shape of the light curve relative to the mass of the ejecta, which demonstrates an increase in curve width with increasing mass. As the initial shock temperature increases, the peak luminosity increases with increasing energy, but has little effect on the shape of the light curve. The number of peaks within the first 300 days of the explosions depends primarily on the ejecta mass density and the energy of the explosion. In general, the second light curve peak is brighter than the shock-breakoutproduced peak, except for a few isolated situations with initial conditions on the lower end of our parameter range. The lowintensity third peak appears in simulations with lower mass combined with higher energy, but this peak disappears with increasing radius and temperature, as it occurs beyond the 300-day mark.

\section{Type IIn Swift UVOT Observations}

The two Swift UVOT-observed SNe, SN 2009ip (Maza et al. 2009; Drake et al. 2012; Prieto et al. 2012, 2013; Margutti et al.2013) and SN 2011ht (Boles et al. 2011; Roming et al. 2012; Humphreys et al. 2012; Mauerhan et al. 2013) were chosen to analyze our suite of models based on the rare capture 

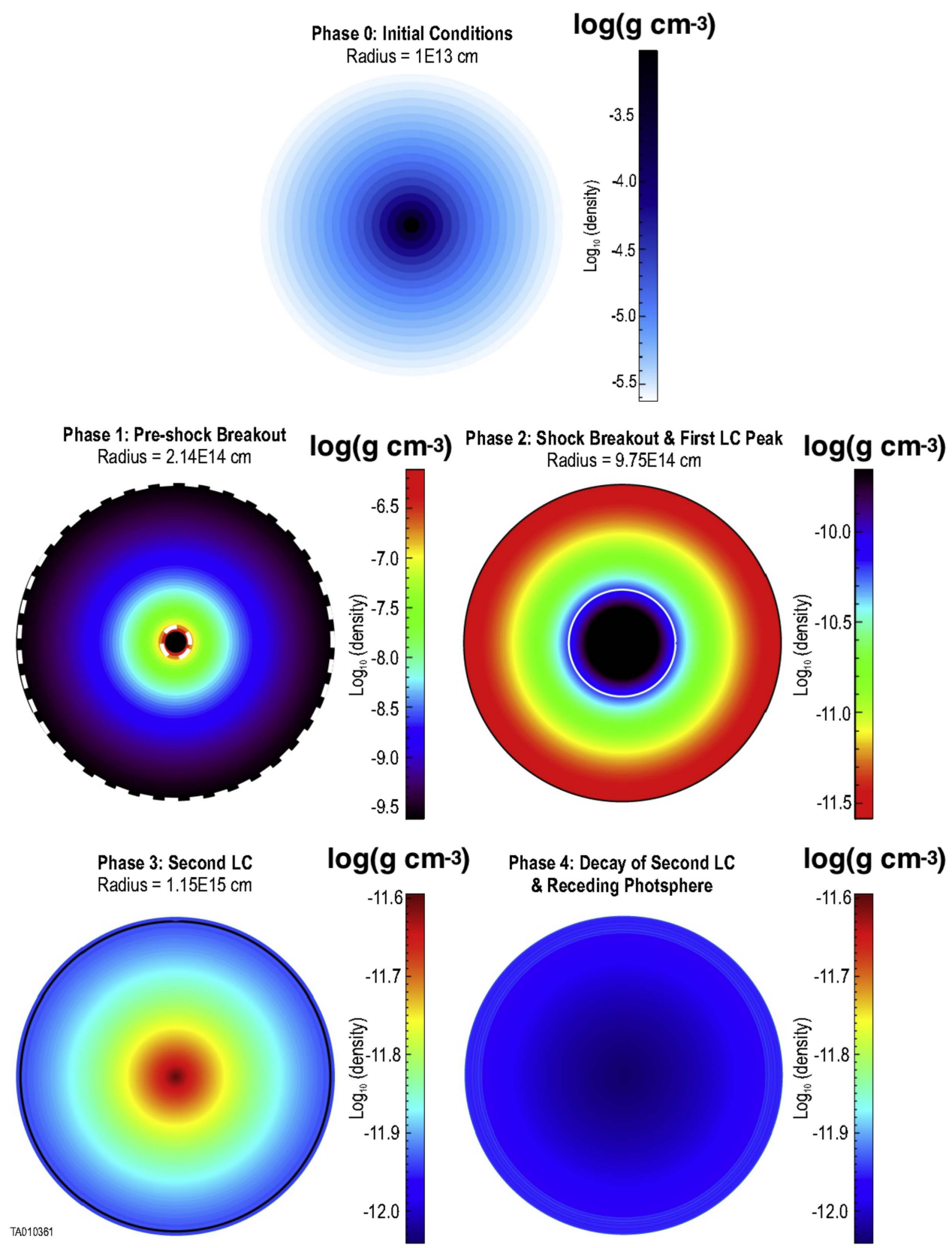

Figure 4. Initial conditions and the four phases of the explosion for $15 M_{\odot}$ ejecta, an initial radius of $1 \times 10^{13} \mathrm{~cm}$,a shock temperature of $1,000,000 \mathrm{~K}$, and $1 \times 10^{51} \mathrm{erg}$ explosion. Phase 0 represents the initial density distribution of the ejecta before explosion, at time $=0$. Phase 1 represents the density of the outer mass layer as it expands during the pre-shock breakout phase. The solid dark inner circle resembles the initial radius of the star (time $=0$ ), and the outer radius is the position of the outer ejecta layer at the end of Phase 1 (time $\approx 5$ days). Phase 2 demonstrates how the density of the outer mass layer changes during shock breakout. The solid black inner region signifies the final radius of Phase 1, and the solid white line represents the first light curve peak. Phase 3 occurs during the second light curve peak. The solid black line represents the second light curve peak. Phase 4 occurs during the decay of the second peak. The radial expansion reaches a maximum at the end of Phase 3, and the interaction region moves inward.

of the initial rise to peak in all six UVOT filters, and also were chosen due to the uniqueness problem revealed in their distinct light curve features. SN 2009ip luminosities rise quickly to the peak luminosity, which is then followed by a fast decay, while SN 2011ht demonstrates a relatively slower rise to the peak, and reveals an extended peak period similar to IIP SNe. By exploring the parameter space in these fast-running simple models, we can analyze which initial parameters contribute to the difference between these two well-studied IIn events. We focus on the UV and optical properties of these events, and produce blackbody trends using a method that compares the samples' observed magnitudes to synthetic SN magnitudes (Pritchard et al. 2014; de la Rosa et al. 2016).

The comparison synthetic magnitudes are generated from blackbody spectra with a blackbody temperature range from $30,000 \mathrm{~K}$ to $1000 \mathrm{~K}$. The SN UVOT observations are compared to the synthetic magnitudes using a chi-squared minimization in order to determined the best-fit blackbody spectra and temperature for each epoch. The blackbody temperatures derived from this technique are used to generate the SN blackbody flux, which is used to calculate the blackbody luminosity at each epoch. The resulting blackbody luminosity 

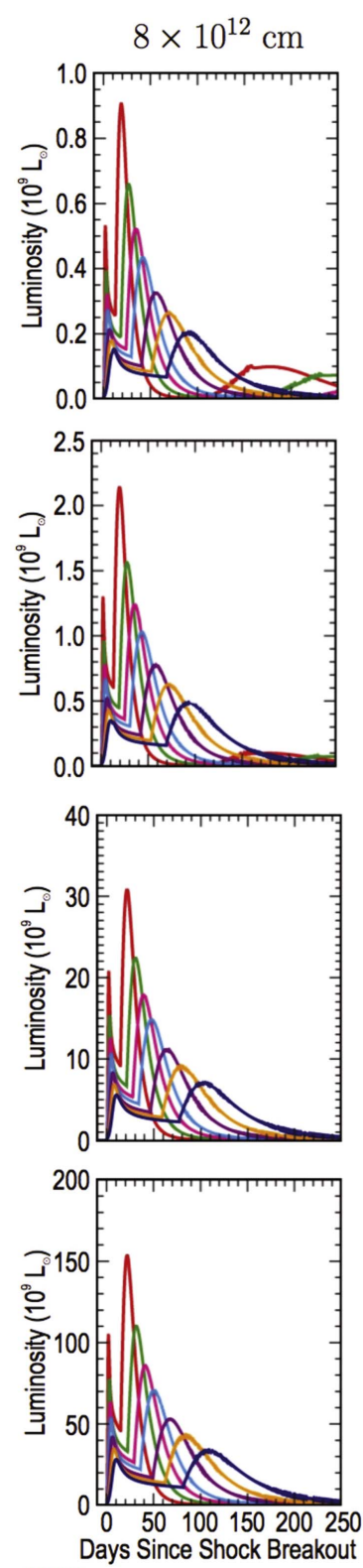

TA010360
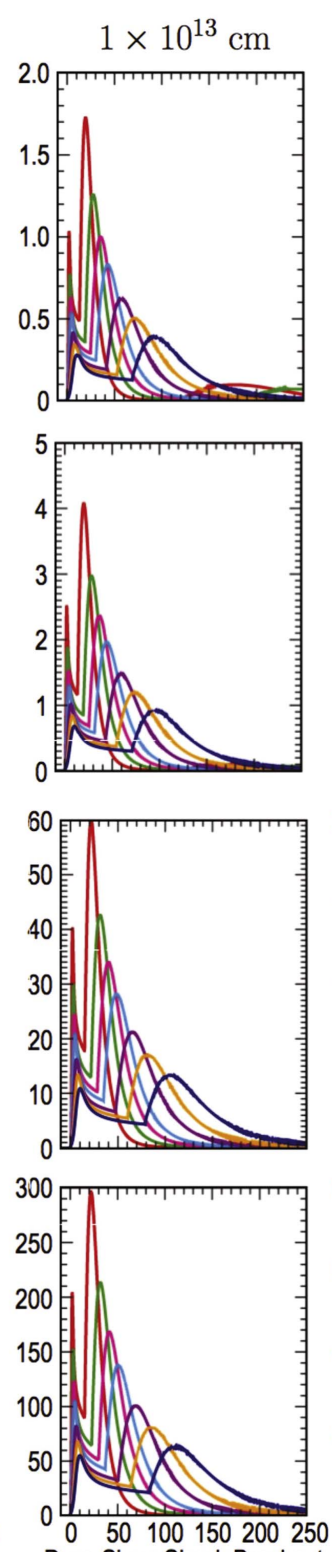

Days Since Shock Breakout
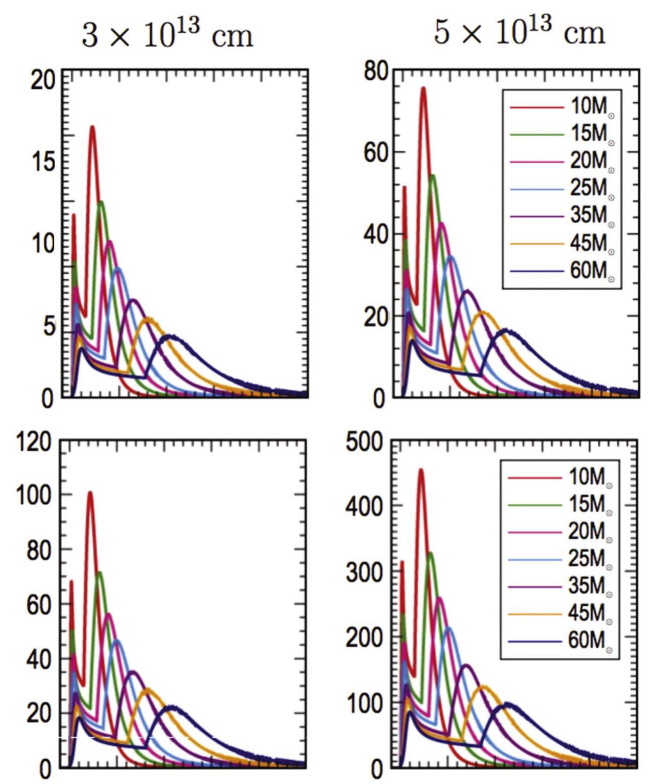

$1 \times 10^{6} \mathrm{~K}$
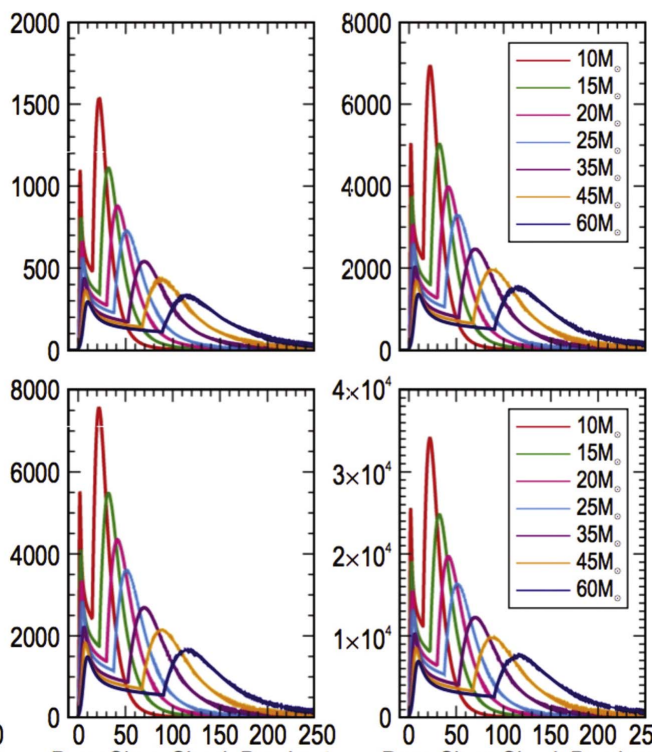

Days Since Shock Breakout

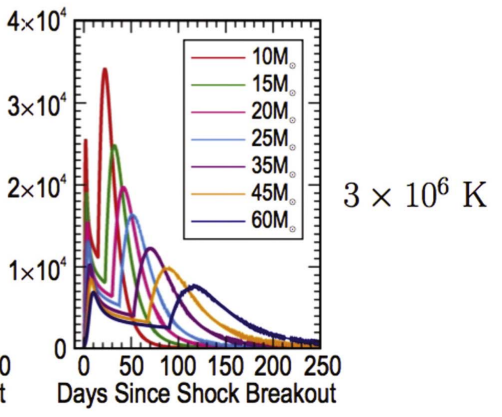

Figure 5. Modeled blackbody luminosity trends for an SN explosion with an energy of $1 \times 10^{51} \mathrm{erg}$. Each column varies by initial radius, starting with the smallest radii modeled on the left $\left(8 \times 10^{12} \mathrm{~cm}\right)$. The red line represents $10 M_{\odot}$ ejecta and the dark blue line represents $60 M_{\odot}$. Each row varies by shock temperature, starting with the lowest temperature simulated $\left(8 \times 10^{5} \mathrm{~K}\right)$ models in the top panel.

trends generated from the SN UV-optical photometry are compared to UVOT-based bolometric luminosity derived from the wavelength range for each UVOT filter. In this study, we generate modeled pseudo UV-optical bolometric light curves using the UVOT filter wavelength range, and focus on peak luminosity and light curve duration. The peak luminosities of SN 2009ip and SN 2011ht produced from this technique are $1.45 \times 10^{43} \mathrm{erg} \mathrm{s}^{-1}$ and $8.42 \times 10^{42} \mathrm{erg} \mathrm{s}^{-1}$, and define the duration of the generated bolometric light curve as the FWHM of the luminosity curves of $20 \pm 5$ days and $60 \pm 24$ days, respectively.

\subsection{UV-optical Luminosities}

We produce UV-optical pseudo-bolometric luminosities from the modeled luminosities, and compare the peak pseudo-bolometric luminosity as a function of its FWHM to the same relationship of SN luminosities produced from UVOT photometry. Our comparison focuses on the first and second modeled luminosities, since the third peak luminosities do not display the desired intensities to produce IIn peak luminosities, and their appearance occurs long after explosion.

Figure 6 demonstrates the peak luminosity of the first peak or the shock breakout peak as function of the FWHM of the curve, compared to the peaks and curve durations of SN 2009ip and SN 2011ht. The increase in shock temperature and energy results in a brighter $\mathrm{SN}$ event, but a lower mass and radius can counteract that relationship and result in a less intensified SN shock breakout curve. The duration of the curve also depends on the mass of the ejecta and the energy. The observed SN luminosity trends fall in an initial temperature range between $1 \times 10^{6} \mathrm{~K}$ and $2 \times 10^{6} \mathrm{~K}$. Due to the short duration of the shock breakout, SN 2011ht fails to match several of the 

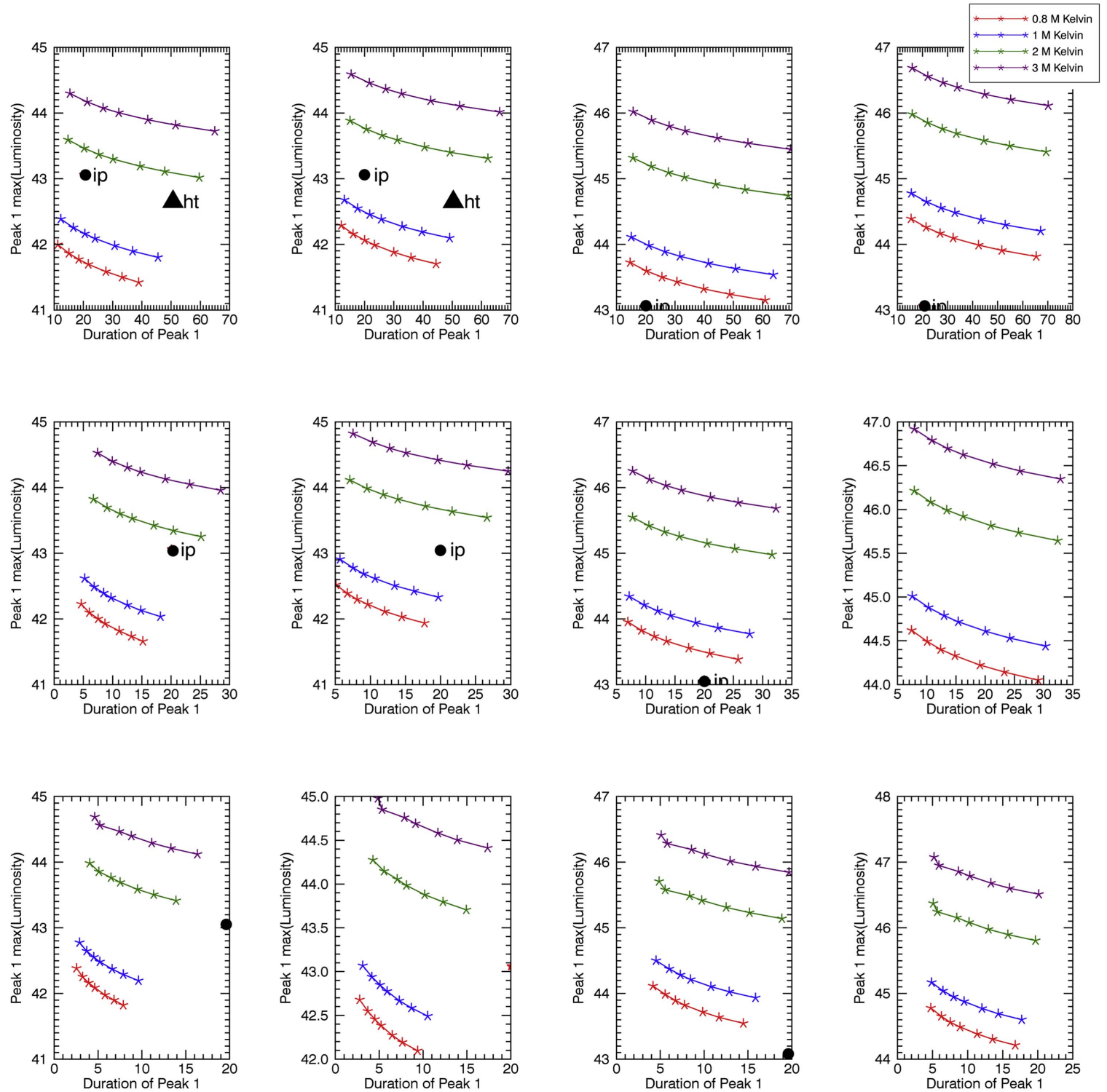

Figure 6. Peak UV-optical bolometric luminosity as function of FWHM duration for first peak in the light curve (shock breakout light curve). Each column varies by initial radius, starting with smallest radii modeled on the left. The black dot represents the peak luminosity and the duration of the SN 2009ip pseudo-bolometric light curve. The black triangle represents the peak luminosity and the duration of the SN $2011 \mathrm{ht}$ pseudo-bolometric light curve. Each color represents a different shock breakout temperature, with purple representing models with a temperature of $3 \times 10^{6} \mathrm{~K}$, and red representing the lowest modeled temperature, $8 \times 10^{5} \mathrm{~K}$.

models, comparable only to models with higher mass and lower energy, in comparison to SN 2009ip, which reveals the potential to match with the lower-mass ejecta and multiple energies.

Figure 7 demonstrates the peak luminosity of the second peak as a function of curve duration, compared to the curve properties of SN 2009ip and SN 2011ht. Analogous to the first peak, the increases in shock temperature, energy, and radius, and the decrease in ejecta mass, result in a brighter event, but the duration of the second curve is longer than the shock-breakoutproduced curve. As with the first peak, the observed SN luminosity trends fall in an initial temperature range between $1 \times 10^{6} \mathrm{~K}$ and $2 \times 10^{6} \mathrm{~K}$, but the comparison results in multiple potential initial conditions. In general, the peak-duration relationship of SN 2009ip represents lower mass/lower radii with higher energies, while SN 2011ht can be narrowed down to a radius of $1 \times 10^{13} \mathrm{~cm}$, with potentially various ejecta masses and explosion energies. The peak-duration relationship of SN 2009ip matched best with lower-mass ejecta models with higher radius, for the two lowest radii modeled. On the other hand, SN 2011ht has the potential to match with ejecta masses from 10-45 $M_{\odot}$, for various energy combinations, but seems to fit best with an initial radius of $1 \times 10^{13} \mathrm{~cm}$. In the following section, we compare a subset of the models presented in the previous sections to the UV and optical light curves of these two SNe.

\section{UV and Optical Light Curves}

We were able to identify possible initial conditions for two well-observed IIn SNe based on their pseudo-bolometric UVoptical luminosity peak and curve duration relationship. We compare the UVOT light curves of the models with similar peaks and curve durations to the $\mathrm{SNe}$ light curves in order to explore which particular set of initial conditions contributes to UV and optical light curve. The modeled UVOT light curves were produced using UVOT filter response curves, and represent these modeled $\mathrm{SNe}$ as being observed by Swift 

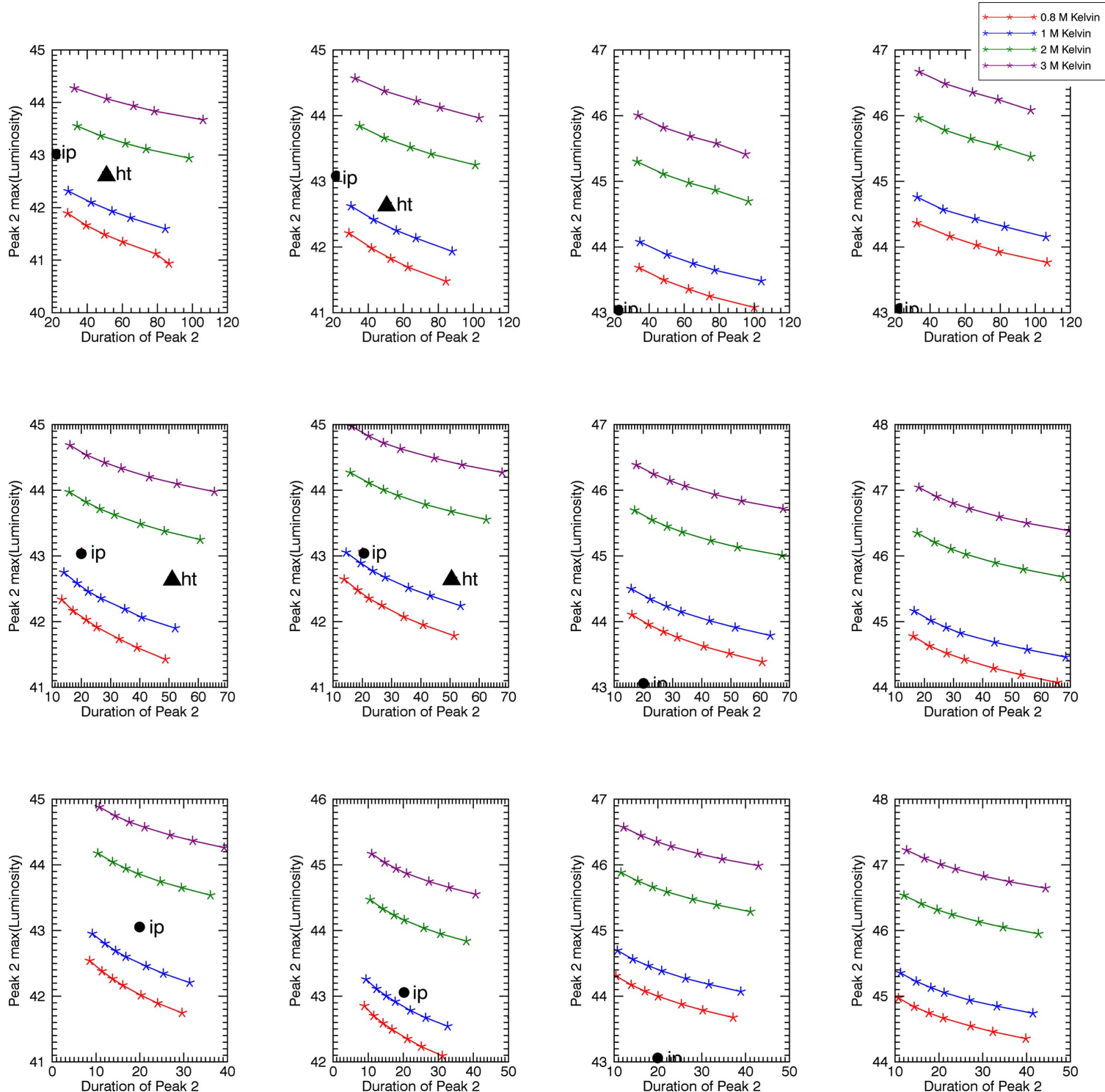

Figure 7. Peak UV-optical bolometric Luminosity as a function of FWHM duration for the second light curve peak (main light curve). Each column varies by initial radius, starting with smallest radii modeled on the left. The black dotted star represents the peak luminosity and the duration of the SN 2009ip pseudo-bolometric light curve. The black triangle represents the peak luminosity and the duration of the SN 2011ht pseudo-bolometric light curve. Each color represents a different shock breakout temperature, with purple representing models with a temperature of $3 \times 10^{6} \mathrm{~K}$, and red representing the lowest modeled temperature, $8 \times 10^{5} \mathrm{~K}$.

UVOT. From the peak and light curve duration comparison, these $\mathrm{SNe}$ coincide best with the models on the lower simulated radius range. The ejecta mass dependence reveals SN 2009ip corresponding to $\mathrm{SN}$ models on the lower mass range, while the light curve peak and duration comparison exhibits a wide range of probable ejecta masses for SN 2011ht. Since the shock temperatures for both these events fall within the same range and temperature has a greater effect on the peak luminosity rather than the shape, all of the simulations analyzed in this section have an initial temperature of $1 \times 10^{6} \mathrm{~K}$.

\subsection{Absolute Magnitudes}

Overall, the SNe UV magnitude matched the peak magnitudes of several models, but failed to match the shape of the light curve due to the short duration of the first peak. Therefore, we focus on the comparisons of the peak and curve shape of the second light curve. We analyze the modeled UV and optical light curves and the UV light curve properties of SN 2009ip and SN 2011ht in Figures 8 and 9.

SN 2009ip: Figure 8 compares UV and optical modeled light curves for low-mass ejecta, with an initial shock temperature of $1 \times 10^{6} \mathrm{~K}$, and initial explosion energies of $1 \times 10^{51}$ for the smallest initial radii $\left(8 \times 10^{12} \mathrm{~cm}\right.$ and $\left.1 \times 10^{13} \mathrm{~cm}\right)$. The peak UV magnitudes produced from the smallest radius, $8 \times 10^{12} \mathrm{~cm}$, are $2.5 \mathrm{mag}$ fainter than those of SN 2009ip. On the other hand, the models with $15 M_{\odot}$ ejecta with larger radii match the peak UV magnitude of SN 2009ip, but fail to match the overall shape of the light curve. The peak optical magnitudes of a $1 \times 10^{13} \mathrm{~cm}$ radius star are brighter than the SN 2009ip optical peak light curve. The optical peak magnitude match for SN 2009ip is $35 M_{\odot}$ ejecta with radii of $8 \times 10^{12} \mathrm{~cm}$.

In order to identify which initial conditions affect the shape of the light curve, we normalized each light curve to its peak absolute magnitude. For a progenitor star with a radius of 

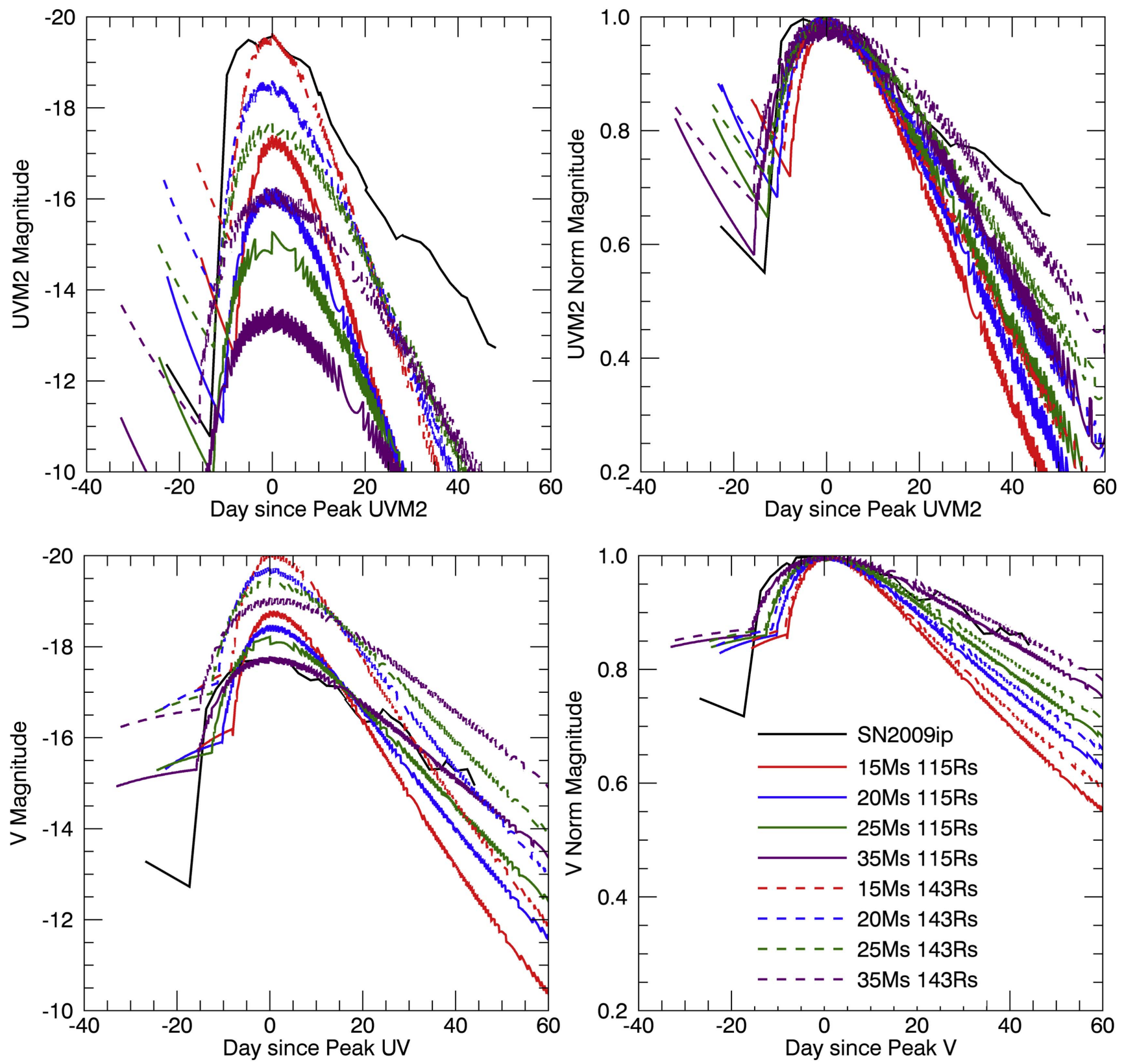

Figure 8. The black line signifies SN 2009ip UV and optical light curves. The solid line corresponds to an initial radius of $1 \times 10^{13} \mathrm{~cm}$ and the dotted lines correspond to a radius of $8 \times 10^{12} \mathrm{erg}$.

$8 \times 10^{12} \mathrm{~cm}$, the rise to the peak matches best with $15 M_{\odot}$. ejecta, while an initial radius of $1 \times 10^{13} \mathrm{~cm}$ matches best with $20 M_{\odot}$ ejecta. The mass of the ejecta corresponding to the rise to the peak is different for each initial radius, but the decay portion of the light curve resembles the trend of an $\mathrm{SN}$ with a higher ejecta mass, $35 M_{\odot}$. Overall, the peak UV magnitude is matched best by a $1 \times 10^{13} \mathrm{~cm}$ radius star, with $15 M_{\odot}$, and an explosion energy of $1 \times 10^{51} \mathrm{erg}$. The shape of the UV light curve depends on the mass of the ejecta, the rise to the peak is best matched by $20 M_{\odot}$, while the decay matches best with $35 M_{\odot}$ ejecta. The optical light curve shape can be modeled by a $1 \times 10^{13} \mathrm{~cm}$ and a $8 \times 10^{12} \mathrm{~cm}$ progenitor star with $35 \mathrm{M}_{\odot}$ ejecta.

SN 2011ht: Figure 9 compares UV and optical modeled light curves for an initial radius of $1 \times 10^{13} \mathrm{~cm}$, with an initial shock temperature of $1 \times 10^{6} \mathrm{~K}$, and initial explosion energies of $1 \times 10^{50}$ and $1 \times 10^{51}$. The peak UV magnitudes could be reproduced by the models with $20 M_{\odot}$ ejecta with an explosion energy of $1 \times 10^{51} \mathrm{erg}$, and the UV light curve shape resembles an $\mathrm{SN}$ with a more massive envelope and a lower explosion energy. The optical light curve properties resemble an explosion energy of $1 \times 10^{50}$, where the peak is produced by $20 M_{\odot}$ ejecta, and the shape of the light curve is the result ofejecta twice as massive.

In comparison to the initial conditions of the $\mathrm{SN}$ ejecta reproducing UV light curve properties, the optical light curve is the product of denser ejecta. For both SNe comparisons, the ejecta producing optical light curves are twice as massive as the ejecta with comparable UV peak magnitudes. Therefore, in order to evaluate the effects of the SN initial conditions on late 

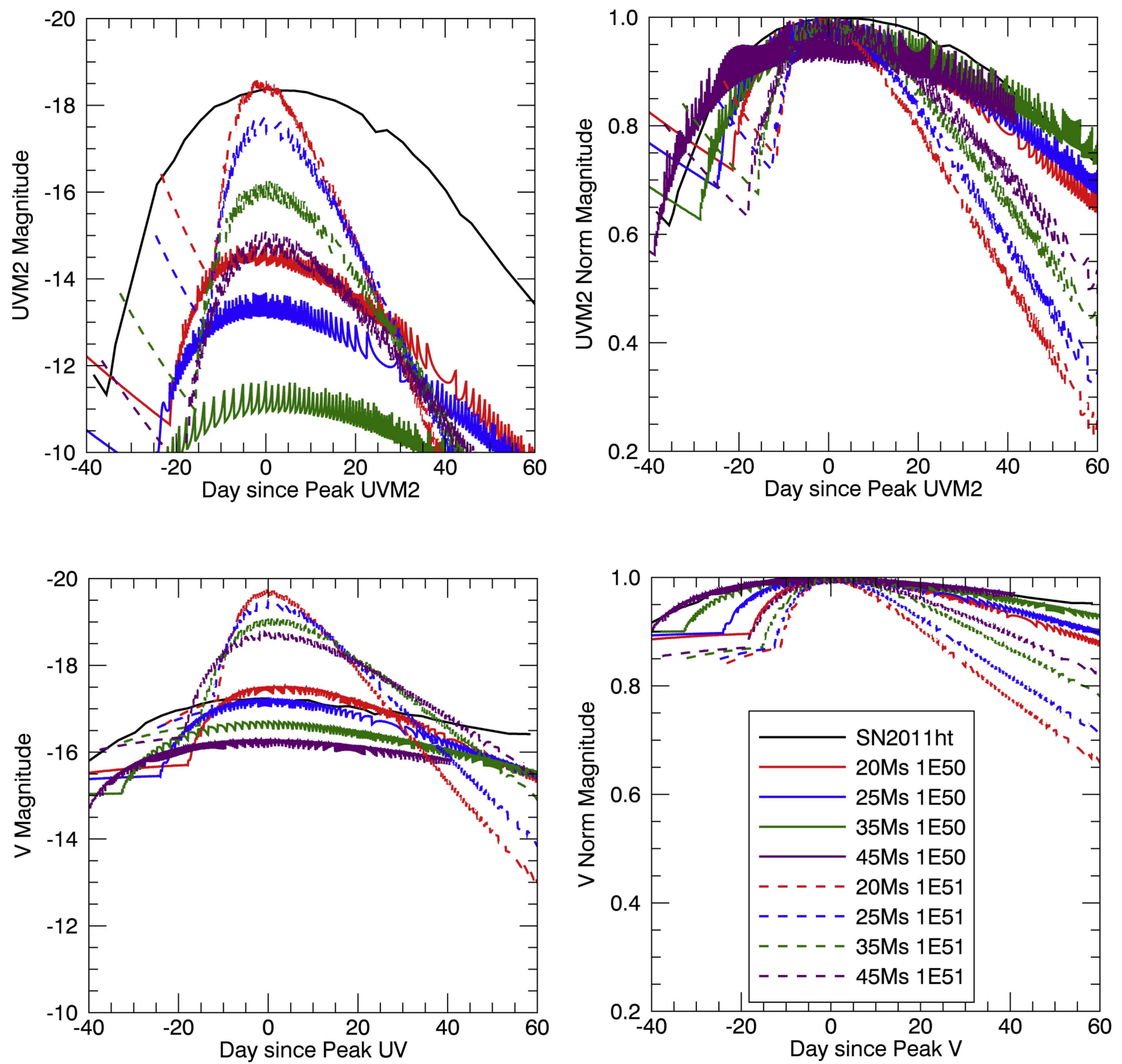

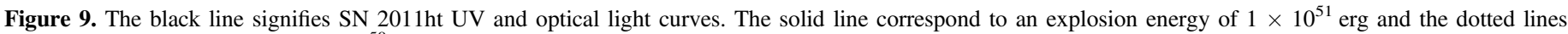
correspond to an explosion of $1 \times 10^{50} \mathrm{erg}$.

time UV/optical emission and a fundamental difference between these IIn events, we compare SN 2009ip and SN 2011ht UV and optical light curves to models with the same initial ejecta mass, $35 M_{\odot}$, but different progenitor radii and explosion energies. In Figure 10, the modeled light curves are shifted in order to match the peak magnitude for each SN light curve. For SN 2009ip, the UV and optical absolute magnitudes are compared to the $\mathrm{SN}$ model with a $8 \times 10^{12} \mathrm{~cm}$ radius and $1 \times 10^{51} \mathrm{erg}$. The modeled UV light curve is shifted up by $6.2 \mathrm{mag}$, whereas the optical light curve is not shifted. For SN $2011 \mathrm{ht}$, the UV and optical absolute magnitudes are compared to the $\mathrm{SN}$ model with a $1 \times 10^{13} \mathrm{~cm}$ radius and explosion energy of $1 \times 10^{50} \mathrm{erg}$. The modeled UV light curve is shifted up by $7.2 \mathrm{mag}$, whereas the optical light curve is shifted up by 0.5 mag.

\section{Discussion}

The simple semi-analytic SN simulations presented can be easily modified to incorporate additional phases of the explosion, and their fast-running capabilities make them an essential tool for exploring the parameter space, and the physical processes of the explosion. One issue with simulating a limited amount of SN cases is the possibility of overlooking any degeneracy. Results from analytic SN simulations are usually compared to their numerically modeled counterparts in order to evaluate the accuracy of the analytically deduced solution. Unfortunately, the computational time needed to run numerical simulations to restore realistic physical properties for the pre-explosion star limits SN studies by only being able to analyze a single $\mathrm{SN}$ event. On the other hand, numerical 
SN2009ip

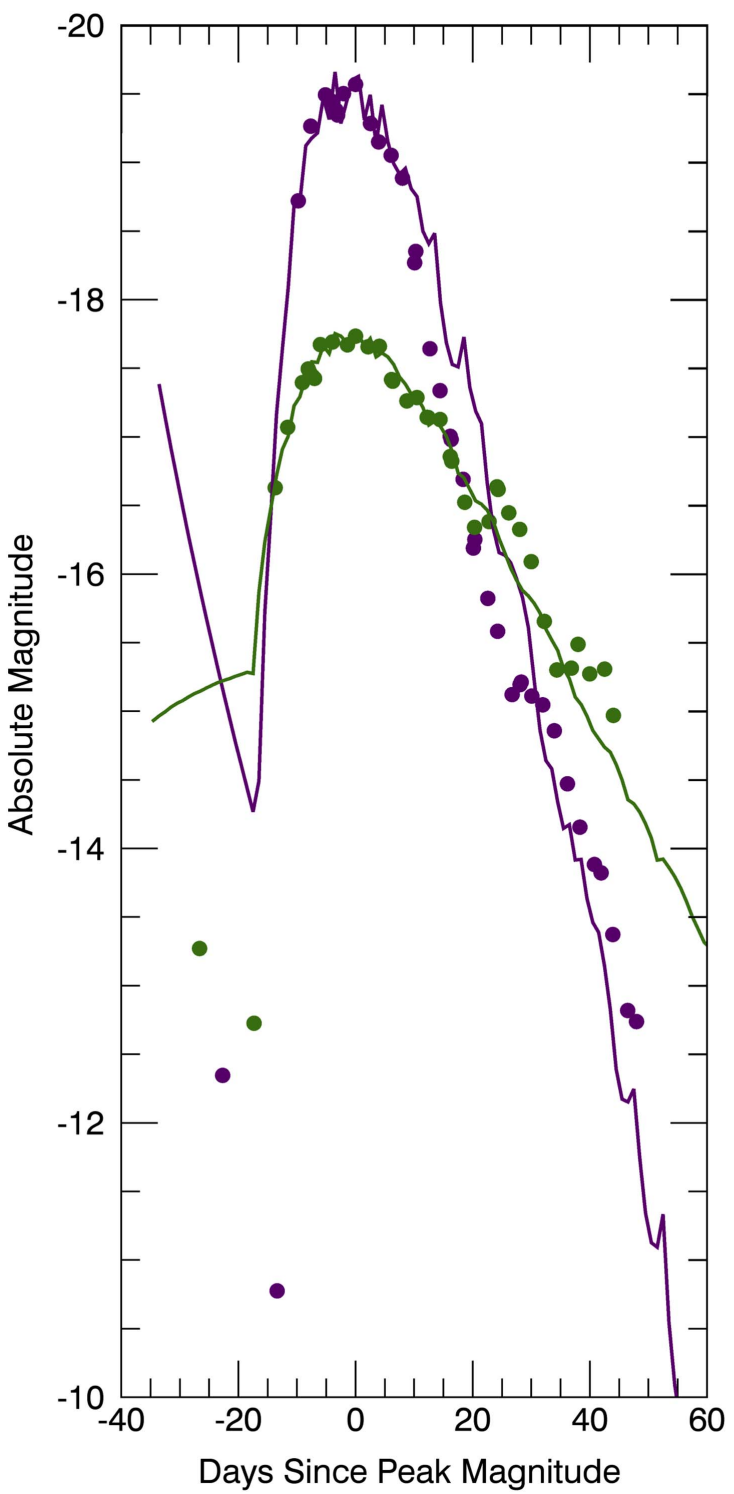

SN2011ht

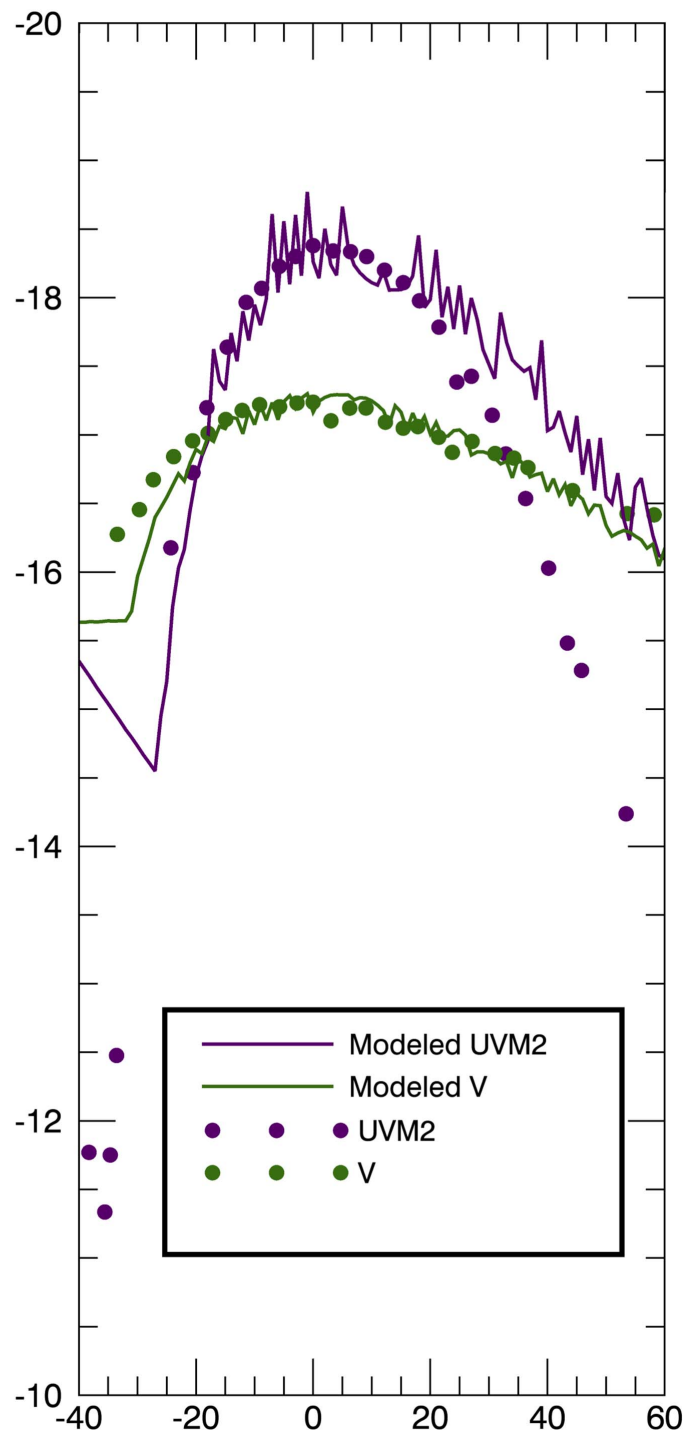

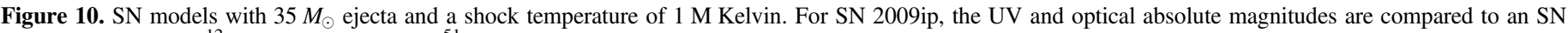

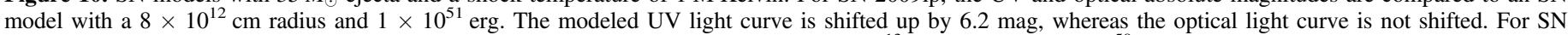

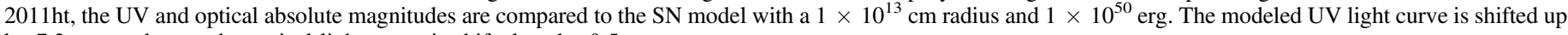
by $7.2 \mathrm{mag}$, whereas the optical light curve is shifted up by $0.5 \mathrm{mag}$.

simulations typically include the phases and transitions of the evolution, starting from the beginning of the main sequence phase up to the point of explosion, which then uses the initial conditions of the progenitor star at the point of explosion to capture the SN evolution up until the end of the nuclear burning phase. These simulations are repeated by varying only a few parameters, until the modeling results fit the peak and decay light curve features of a particular SN observation. The input parameter for the best-fit model (e.g., the energy of the explosion, the mass of the progenitor star, and the radius of the star) are considered the general properties of the progenitor star and explosion. Therefore, simple analytic calculations can be used to complement more realistic numerical simulations by providing any missing physical processes associated with the explosion mechanism, along with the significance of the stellar and explosion parameters.
Based on the modeling technique presented in this paper, we simulate various SN initial conditions starting from the time the shock reaches the outermost shock-heated ejecta at a given radius (which we approximate to time of explosion), to 300 days after explosion. Depending on the initial conditions of the simulation, several of the models revealed a three-peak structure, i.e., lower-mass, higher-energy simulations. Further analysis of the three-peak structure suggests three distinct energy sources: shock breakout, receding photosphere, Ni-56 decay. Each peak displayed unique light curve characteristics, and the light curve's dependence on the initial conditions of the explosion.

The first peak light curve occurs as the outer mass layer breaks out of the inner photospheric region. During this SN phase, UV production dominates over redder wavelengths, but its light curve shape resembles a pulse more than a full light curve as the 
initial explosion energy increases. As the outer mass layer diffuses, it is no longer a sufficient energy source, and the photon production site shifts to the following optically denser inner layer, initiating the rise of the second light curve. The transition into the second light curve peak depends on the density of the outer layer of the star, and the energy of the explosion. Lowenergy explosions with a higher density profile star experience a delayed transition relative to a more energetic explosion for a larger, less massive star.

The rise to the second light curve peak is dominated by the remaining inner mass layers, which quickly diffuse as the explosions drive material away from the star. The decay portion of the second light curve is powered by the denser layers approaching as the dense core region is uncovered. The transition of the energy source occurs days after the peak luminosity, and varies based on the initial density profile of the star and the explosion energy. Once the interaction radius has reached a maximum, the photosphere begins to recede inward, and the innermost ejecta are responsible for photon production. The transition to the ${ }^{56} \mathrm{Ni}$ decay-powered light curve in our models is revealed as a long tail-like structure after the main light curve decay. This tail-like portion of the second light curve continues until the innermost mass layer, i.e., the core, becomes the dominant source, producing the third peak. From our suite of models, the appearance of the third peak within 300 days of the explosion depends on the density profile, the explosion energy, and the initial temperature.

Based on the peak-duration trends, we select a smaller sample of models to compare with SN UVOT light curves. The observation and modeling comparison reveals that the initial shock temperature falls between 1,000,000 K and 2,000,000 K. The peak-duration ratio of SN 2009ip matched best with lowermass, 10-25 $M_{\odot}$, ejecta models for the two lowest radii modeled, and an explosion of $1 \times 10^{51} \mathrm{erg}$. On the other hand, SN 2011ht potentially matches with ejecta masses from 10-45 $M_{\odot}$, for various energy combinations, and fits best with an initial radius of $1 \times 10^{13} \mathrm{~cm}$. In general, UV light curve shape and optical light curve properties are produced from denser ejecta relative to the ejecta properties producing UV peak magnitudes. For both comparison $\mathrm{SNe}$, the ejecta masses of models capable of resembling the UV and optical light curve shape were twice as massive as the models with the bestmatched UV peak, and produced UV peak magnitudes 7 mag fainter relative to the observed SNe. The difference in the production of UV and optical peak magnitudes could arise from different energy sources and production sites. The initial rise in UV magnitudes is the product of the energy deposited from the post-shock breakout process, i.e., the shock heating into the remaining hydrogen envelope, while less intense optical emission arises from the energy deposited into denser inner stellar material. Our modeling approach suggests the difference between these two events is the density of the progenitor star and the energy of the explosion. SN 2009ip is the result of a denser progenitor star with a higher explosion energy relative to SN $2011 \mathrm{ht}$.

\section{Conclusions}

In recent years, due to the uncertainty behind the types of massive star that evolve into different types of core-collapse events, there has been an increase in core-collapse supernova surveys aiding the advancement of numerical supernova simulations that explore the properties of the star before the explosion. The characteristics of IIn SN light curves have been analytically and numerically analyzed by several studies. The fast-running simulations presented here allow us to explore the $\mathrm{SN}$ parameter space, analyze the energy source driving IIn light curves, and interpret the effects of initial conditions on light curve characteristics, light curve shape (rise and decay), and peak luminosities. With these models, we can explore the questionable heterogeneous nature of IIn SNe by identifying the similarities and differences in their explosion process, and evaluating which parameters are potentially causing that diversity, while also searching for missing physical processes in order to assist the evolution of more realistic numerical simulations.

\section{ORCID iDs}

Pete Roming (ib https://orcid.org/0000-0002-5499-953X Chris Fryer (iD https://orcid.org/0000-0003-2624-0056

\section{References}

Arnett, W. D. 1980, ApJ, 237, 541

Arnett, W. D. 1982, ApJ, 253, 785

Arnett, W. D., Fryer, C. L., \& Matheson, T. 2016, arXiv:1611.08746

Bersten, M. C., Benvenuto, O., \& Hamuy, M. 2011, ApJ, 729, 61

Blinnikov, S. I., \& Bartunov, O. S. 1993, A\&A, 273, 106

Boles, T., Pastorello, A., Stanishev, V., et al. 2011, CBET, 2851, 1

Chatzopoulos, E., Wheeler, J. C., \& Vinko, J. 2012, ApJ, 746, 121

Chevalier, R. A. 1992, ApJ, 394, 599

de la Rosa, J., Roming, P., Pritchard, T., \& Fryer, C. 2016, ApJ, 820, 74

Dessart, L., Hillier, D. J., Li, C., \& Woosley, S. 2012, MNRAS, 424, 2139

Drake, A. J., Howerton, S., McNaught, R., et al. 2012, ATel, 4334, 1

Filippenko, A. V. 1997, ARA\&A, 35, 309

Frey, L. H., Even, W., Whalen, D. J., et al. 2013, ApJS, 204, 16

Frey, L. H., Fryer, C. L., \& Young, P. A. 2013, ApJL, 773, L7

Fryer, C. L. 2009, ApJ, 699, 409

Fryer, C. L., Ruiter, A. J., Belczynski, K., et al. 2010, ApJ, 725, 296

Gal-Yam, A., Leonard, D., Fox, D., et al. 2007, ApJ, 656, 372

Gehrels, N., Chincarini, G., Giommi, P., et al. 2004, ApJ, 611, 1005

Humphreys, R. M., Davidson, K., Jones, T. J., et al. 2012, ApJ, 760, 93

Kozyreva, A., Gilmer, M., Hirschi, R., et al. 2017, MNRAS, 464, 2854

Liu, Y.-Q., Modjaz, M., Bianco, F. B., \& Graur, O. 2016, ApJ, 827, 90

Margutti, R., Milisavljevic, D., Soderberg, A., et al. 2013, arXiv:1306.0038

Mauerhan, J. C., Smith, N., Silverman, J. M., et al. 2013, MNRAS, 431, 2599

Maza, J., Hamuy, M., Antezana, R., et al. 2009, CBET, 1928, 1

Moriya, T. J., Maeda, K., Taddia, F., et al. 2013, MNRAS, 435, 1520

Moriya, T. J., Pruzhinskaya, M. V., Ergon, M., \& Blinnikov, S. I. 2016, MNRAS, 455, 423

Morozova, V., Piro, A. L., Renzo, M., et al. 2015, ApJ, 814, 63

Prieto, J. L., Brimacombe, J., Drake, A., \& Howerton, S. 2013, ApJL, 763, L27

Prieto, J. L., Brimacombe, J., \& Drake, A. J. 2012, ATel, 4439, 1

Pritchard, T., Roming, P., Brown, P. J., Bayless, A. J., \& Frey, L. H. 2014, ApJ, 787, 157

Roming, P., Pritchard, T., Prieto, J., et al. 2012, ApJ, 751, 92

Roming, P. W., Kennedy, T. E., Mason, K. O., et al. 2005, SSRv, 120, 95

Schlegel, E. M. 1990, MNRAS, 244, 269

Smith, N., Li, W., Filippenko, A. V., \& Chornock, R. 2011, MNRAS, 412, 1522

Valenti, S., Howell, D. A., Stritzinger, M. D., et al. 2016, MNRAS, 459, 3939 